\title{
Note \\ Organocatalytic Site-Selective Acylation of 10-Deacetylbaccatin III
}

\author{
Masanori Yanagi, ${ }^{a}$ Ryo Ninomiya, ${ }^{a}$ Yoshihiro Ueda, ${ }^{a}$ Takumi Furuta, ${ }^{a}$ Takeshi Yamada,${ }^{b, c}$ \\ Toshiaki Sunazuka, ${ }^{b, c}$ and Takeo Kawabata ${ }^{*, a}$ \\ ${ }^{a}$ Institute for Chemical Research, Kyoto University; Uji, Kyoto 611-0011, Japan: ${ }^{b}$ Kitasato Institute \\ for Life Sciences, Kitasato University; 5-9-1 Shirokane, Minato-ku, Tokyo 108-8641, Japan: and \\ ${ }^{c}$ Graduate School of Infection Control Sciences, Kitasato University; 5-9-1 Shirokane, Minato-ku, \\ Tokyo 108-8641, Japan. \\ Received January 13, 2016; accepted January 18, 2016; advance publication released online February 23, 2016
}

\begin{abstract}
Organocatalytic site-selective diversification of 10-deacetylbaccatin III, a key natural product for the semisynthesis of taxol, has been achieved. Various acyl groups were selectively introduced into the $\mathrm{C}(10)-\mathrm{OH}$ of 10-deacetylbaccatin III. The $\mathrm{C}(10)-\mathrm{OH}$ selective acylation was also applied to acylative site-selective dimerization of 10-deacetylbaccatin III to provide the structurally defined dimer.
\end{abstract} ization

Key words organocatalyst; site-selectivity; acylation; taxane; 10-deacetylbaccatin III; site-selective dimer-

Natural products have been utilized as the most productive candidates for controlling the cellular events and developing new therapeutic agents. ${ }^{1}$ Site-selective (de)functionalization of biologically active natural products was expected to be a useful protocol for further investigating the biological events and the medicinal application. Enzymatic site-selective acylation of complex natural products has been explored to provide derivatives with improved activity and pharmacokinetic properties. ${ }^{2}$ For a beautiful example, enzymatic site-selective acylation of immunosuppressive rapamycin at the $\mathrm{C}(42)-\mathrm{OH}$ enabled to convert to an antitumor drug, temsirolimus, in onestep. ${ }^{3)}$ Alternatively, site-selective modification of complex natural products by non-enzymatic protocol has also attracted increasing attention, because it enables to provide natural product derivatives with structural diversity and regiochemical profile different from those obtained by enzymatic protocol. ${ }^{4}$ Site-selective acylation of natural products by non-enzymatic methods, however, often encounter difficulties in controlling the site-selectivity due to the structural complexity and the functional group diversity found in the natural substances. Under these backgrounds, we have developed a method for a non-enzymatic site-selective acylation of polyol compounds such as natural glycosides catalyzed by 4-dialkylaminopyridine-type nucleophilic organocatalyst 6 and the derivatives ${ }^{5-10)}$ (Fig. 1). For example, catalyst $\mathbf{6}$ could deliver an acyl group at the $\mathrm{C}\left(4^{\prime \prime \prime \prime}\right)-\mathrm{OH}$ of lanatoside $\mathrm{C}(5)$ in $86 \%$ site-selectivity out of eight potentially reactive hydroxy groups. This siteselectivity seems independent from the intrinsic reactivity of 5 itself, because 4-dimethylaminopyridine (DMAP)-catalyzed acylation provided the corresponding $3^{\prime \prime \prime \prime}-O$-acylate in $97 \%$ site-selectivity. ${ }^{8)}$ Thus, introduction of the acyl group at the $\mathrm{C}\left(4^{\prime \prime \prime \prime}\right)-\mathrm{OH}$ was performed by catalyst-controlled manner and that at $\mathrm{C}\left(3^{\prime \prime \prime \prime}\right)-\mathrm{OH}$ was done by substrate-controlled manner. In the course of our continuous efforts for site-selective diversification of polyol natural products, we found that catalyst
6 promoted highly site-selective acylation of 10 -deacetylbaccatin III (1) at the $\mathrm{C}(10)-\mathrm{OH}$, which is a natural terpenoid available in relatively abundant amount and used as a key intermediate for clinically widely used antitumor agents, taxol (4) and taxotere (3) (Fig. 1). The present site-selective acylation was applied to direct acylative dimerization of $\mathbf{1}$ to successfully provide structurally defined dimer of $\mathbf{1}$ connected by ester linkage at each of the $\mathrm{C}(10)-\mathrm{OH}$ group.

Because of limited amount of taxol (4) available from natural resources, much efforts have been devoted to develop methods for efficient semisynthesis of $\mathbf{4}$ from the abundantly available taxane $\mathbf{1}^{11)}$ Reported semisynthesis commenced from the differentiation of the $\mathrm{C}(7)-\mathrm{OH}$ and $\mathrm{C}(10)-\mathrm{OH}$ with similar reactivity. The relative reactivity of the four hydroxy groups of $\mathbf{1}$ toward acetic anhydride in pyridine has been studied by Guéritte-Voegelein and co-workers, and suggested to be in the order of $\mathrm{C}(7)-\mathrm{OH}>\mathrm{C}(10)-\mathrm{OH}>\mathrm{C}(13)-\mathrm{OH}>\mathrm{C}(1)-$ $\mathrm{OH}^{12)}$ Greene reported $\mathrm{C}(7)-\mathrm{OH}$-selective silylation by making use of the subtle difference in the reactivities of the hydroxy groups, which enabled a highly efficient semisynthesis of $4{ }^{13)}$ Holton found that acylation took place at the $\mathrm{C}(10)-\mathrm{OH}$ selectively using excess amount of acetic anhydride and Lewis acids $\left(\mathrm{ZnCl}_{2}, \mathrm{CeCl}_{3}\right)$ in the absence of pyridine to achieve selective synthesis of baccatin III (2). ${ }^{14,15)}$ Under these backgrounds, we initiated our study to investigate site-selecivity in acylation of $\mathbf{1}$ with catalyst $\mathbf{6}$ and the related catalyst 7-10 (Table 1).

\section{Results and Discussion}

Site-selectivity in acylation of $\mathbf{1}$ was investigated with $\mathbf{6 - 1 0}$ and also DMAP for the control experiment (Table 1). Acylation catalyzed by DMAP with isobutyroyl chloride at $-20^{\circ} \mathrm{C}$ took place preferentially at $\mathrm{C}(10)-\mathrm{OH}$ of $\mathbf{1}$ (entry 1). Use of isobutyric anhydride instead of isobutyroyl chloride resulted in the higher yield and the similar site-selectivity (entry 2). ${ }^{16)}$ 


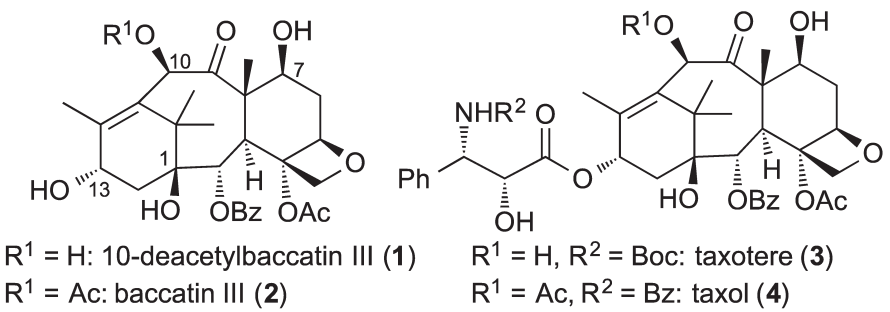<smiles>CCOC(=O)C(Cc1c[nH]c2ccccc12)NC(=O)[C@@H]1CCC(C(=O)N[C@@H](Cc2c[nH]c3ccccc23)C(=O)OCC)N1c1ccncc1</smiles>

$\mathrm{R}^{1}=$ Ac: baccatin III (2)

$\mathrm{R}^{1}=\mathrm{Ac}, \mathrm{R}^{2}=\mathrm{Bz}: \operatorname{taxol}(\mathbf{4})$

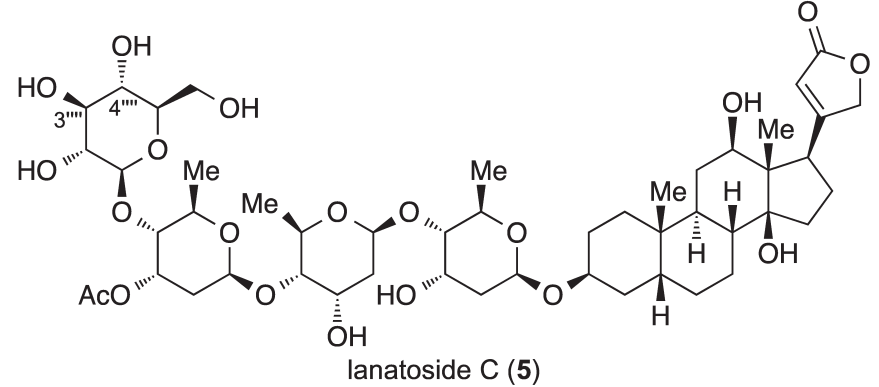

Fig. 1. Structures of 10-Deacetylbaccatin III (1), Baccatin III (2), Taxotere (3), Taxol (4), and Lanatoside C (5)

Catalyst $\mathbf{6}$ useful for site-selective acylation of $\mathbf{1}$ and $\mathbf{5}$.

Table 1. Site Selectivity Profile in Acylation of 10-Deacetylbaccatin III (1)

Site-selectivity

a) $\mathrm{N}, \mathrm{N}$-Diisopropylethylamine (DIPEA) (1.5 eq.) was used instead of collidine.<smiles></smiles>

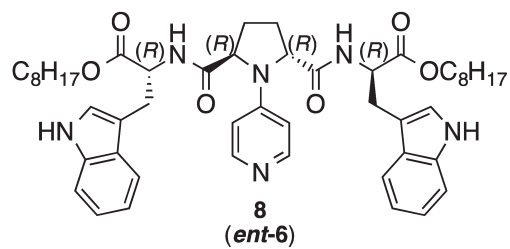<smiles></smiles>

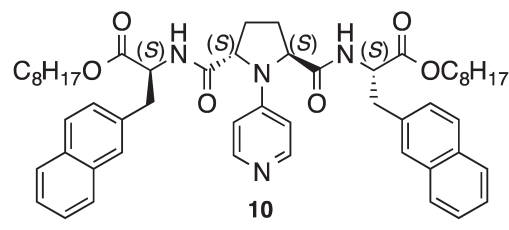


Table 2. Site-Selective Acylation of $\mathbf{1}$ with Various Acid Anhydrides

\begin{tabular}{|c|c|c|c|c|}
\hline & (c) & $\begin{array}{c}6(10 \mathrm{~mol} \%) \\
(\mathrm{RCO})_{2} \mathrm{O}(1.1 \text { eq. }) \\
\text { collidine }(1.5 \text { eq. })\end{array}$ & i-O-acylate & $\widehat{A O}_{\mathrm{Ac}}$ \\
\hline Entry & $\mathrm{R}$ & Time (h) & Monoacylate (\%) & Site selectivity $(7-O: 10-O)$ \\
\hline 1 & $\mathrm{Me}$ & 12 & 99 & $10: 90$ \\
\hline 2 & $\mathrm{C}_{11} \mathrm{H}_{23}$ & 120 & 75 & $8: 92$ \\
\hline $3^{a)}$ & $\mathrm{Ph}$ & 168 & 84 & $5: 95$ \\
\hline 4 & (E)-Ph-CH $=\mathrm{CH}$ & 72 & 93 & $3: 97$ \\
\hline 5 & 2-Thiophenyl & 120 & 75 & $3: 97$ \\
\hline 6 & 3-Furyl & 120 & 85 & $6: 94$ \\
\hline $7^{b)}$ & $\mathrm{CCl}_{3}$ & 1 & 52 & $>99:<1$ \\
\hline
\end{tabular}

a) $\mathrm{Bz}_{2} \mathrm{O}\left(5.0\right.$ eq) and collidine (7.5 eq) were used. b) $\left(\mathrm{CCl}_{3} \mathrm{CO}\right)_{2} \mathrm{O}(3.0 \mathrm{eq})$ and collidine $(5.0 \mathrm{eq})$ were used.

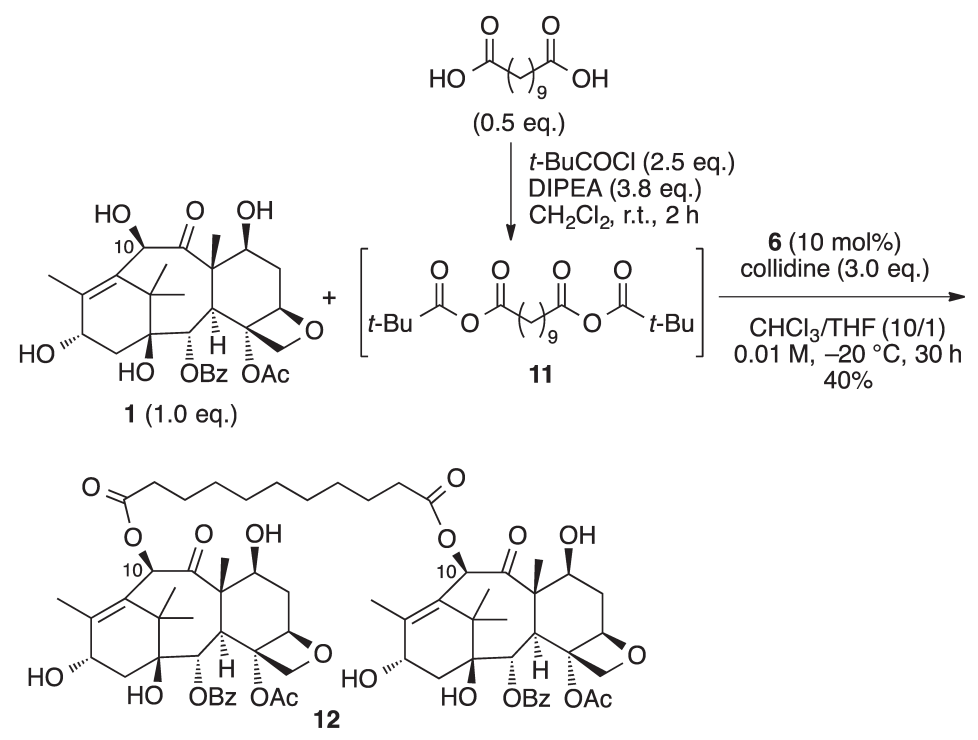

Fig. 2. Direct Site-Selective Dimerization of 10-Deacetylbaccatin III (1) by Catalytic C(10)-OH Selective Acylation

In acylation of $\mathbf{1}$ with isobutyroyl chloride, catalyst $\mathbf{6}$ showed selectivity profile quite similar to that with DMAP (entries $1,3)$. On the other hand, acylation with isobutyric anhydride in the presence of catalyst 6 took place at $\mathrm{C}(10)-\mathrm{OH}$ in high selectivity to provide $10-O$-acylate $\mathbf{1 b}$ in $81 \%$ yield $(93 \%$ siteselectivity among the monoacylates obtained in a combined yield of $87 \%$ : entry 4 ). These results suggest that the carboxylate counter anion of the acylpyridinium intermediate generated from the anhydride and $\mathbf{6}$ may be critically involved in the molecular recognition process of the substrate $\mathbf{1}$ by catalyst 6. ${ }^{17)}$ While $10-O$-acylate $\mathbf{1 b}$ was the major product throughout the acylation reactions promoted by catalysts $\mathbf{6}-\mathbf{1 0}$, the degree of the site-selectivity and the reaction rates were found to be dependent on both the functionalities and the stereochemistry of the side chains of catalysts 6-10 (entries 4-8). Considering that 6 is the best catalyst for the selective $\mathrm{C}(10)-\mathrm{OH}$ acylation of 1, temperature effects were investigated (entries 4, 9-11). At the lower reaction temperature, site-selectivity was slightly increased and the highest selectivity (95\%) was observed at $-40^{\circ} \mathrm{C}$ (entry 11). The reaction in polar solvents (tetrahydrofuran (THF), $N, N$-dimethylformamide (DMF)) provided $\mathbf{1 b}$ with decreased site-selectivity for acylation of the $\mathrm{C}(10)-\mathrm{OH}$ (entries 12, 13). In acylation with catalyst 6 in DMF, the siteselectivity was similar to that observed in acylation catalyzed by DMAP (entries 2 vs. 13). The reaction in DMF proceeded in a quite sluggish manner even in the presence of catalyst 6 (entries 4 vs. 13). All of these results suggest that H-bonding interaction between $\mathbf{1}$ and $\mathbf{6}$ may be responsible for the accelerative and site-selective acylation of the $\mathrm{C}(10)-\mathrm{OH}$.

Introduction of various acyl groups into $\mathrm{C}(10)-\mathrm{OH}$ was performed under the optimum conditions shown in entry 4 in Table 1 (Table 2). Treatment of $\mathbf{1}$ with acetic anhydride in the presence of catalyst $\mathbf{6}$ selectively provided baccatin III (2) in $89 \%$ yield (entry 1). Thus, one-step conversion of $\mathbf{1}$ into $\mathbf{2}$ was achieved by organocatalytic site-selective acylation. Siteselective lipidation of $\mathbf{1}$ was also performed with lauric anhydride (entry 2). Aromatic acyl groups including heteroaromatic groups were also selectively introduced into $\mathrm{C}(10)-\mathrm{OH}$ of $\mathbf{1}$ (entries 3-6). Contrary to these results, the complete reversal in the site-selectivity was observed in acylation with trichloroacetic anhydride, providing the 7-O-acylate exclusively in $52 \%$ yield (entry 7 ). There have been several reports that 
trichloroacetylation of $\mathbf{1}$ took place at $\mathrm{C}(7)-\mathrm{OH} .^{18,19)}$ These results suggested that $\mathrm{C}(10)-O$-acylation with acid anhydride except for trichloroacetic anhydride was performed in a catalyst-controlled manner by virtue of catalyst $\mathbf{6}$ and the $\mathrm{C}(7)-O$-acylation with trichloroacetic anhydride was done in a substrate-controlled manner.

The observed high site-selectivity of acylation catalyzed by $\mathbf{6}$ prompted us to examine direct dimerization of $\mathbf{1}$ by site-selective diacylation (Fig. 2). Dimerization of bioactive compounds is potentially useful strategy toward the discovery of the agents with improved activity profiles including promising activity against the native-compound-resistant infections and cancers. ${ }^{20-25)}$ It was expected that highly site-selective acylation promoted by catalyst $\mathbf{6}$ seemed suitable for acylative dimerization of $\mathbf{1}$ to provide structurally defined dimer of 1. Treatment of $\mathbf{1}$ with mixed anhydride ${ }^{26)} \mathbf{1 1}$ prepared from 0.5 eq of undecanedioic acid and pivaloyl chloride in the presence of 6 gave 10-deacetylbaccatin III dimer 12 in 40\% yield (Fig. 2). Thus, regioisomerically pure 10-deacetylbaccatin III dimer was obtained in one-step from 1 . This protocol may be potentially applicable toward the discovery of new anticancer agents because previous structure-activity relationship studies of taxol (4) revealed that acyl group of $\mathrm{C}(10)-\mathrm{OH}$ was not significantly involved in the main pharmacophore of $4 .^{11,27)}$

In summary, we have developed a method for organocatalytic site-selective acylation of 10-deacetylbaccatin III (1). The $\mathrm{C}(10)-\mathrm{OH}$ selective acylation was successfully applied for direct acylative dimerization of $\mathbf{1}$, which is expected to become an convenient access to a new class of structurally defined and diverse taxol derivatives.

\section{Experimental}

General Remarks ${ }^{1} \mathrm{H}-\mathrm{NMR}$ spectra were measured in $\mathrm{CDCl}_{3}$ solution (tetramethylsilane (TMS): $0.00 \mathrm{ppm}$ or $\mathrm{CHCl}_{3}$ : $7.26 \mathrm{ppm})$ using JEOL ECA-400 $(400 \mathrm{MHz})$ spectrophotometer, unless otherwise stated. ${ }^{13} \mathrm{C}-\mathrm{NMR}$ spectra were measured in $\mathrm{CDCl}_{3}$ solution and referenced to $\mathrm{CDCl}_{3}(77.0 \mathrm{ppm})$ using JEOL ECA-400 (100 MHz) spectrophotometer, unless otherwise noted. IR spectra were recorded on JASCO FT/ IR-4200 spectrometer. Mass spectra were obtained on JEOL JMS-700 mass spectrometer. Optical rotations were measured with JASCO P-2200. Purification of the reaction products was carried out by flash chromatography using Silica Gel (SiliaFlash ${ }^{\circledR}$ F60). Thin layer chromatography was performed on precoated plates $(0.25 \mathrm{~mm}$, silica gel Merck Kieselgel 60F245), and compounds were visualized with UV light and $p$-anisaldehyde stain followed by heating. Preparative thin layer chromatography was performed on precoated plates $(0.5 \mathrm{~mm}$, silica gel Merck Kieselgel 60F245) and visualized with UV light. Preparative HPLC was performed with recycling HPLC system, which was equipped with COSMOSIL 5SL-II column $(20 \times 250 \mathrm{~mm})$ (flow: $4 \mathrm{~mL} / \mathrm{min}$, detection: $254 \mathrm{~nm}$ ). Melting points were measured with Yanaco MICRO MELTING POINT APPARATUS. $\mathrm{CHCl}_{3}$ was purchased from Kanto Kagaku and pre-treated with neutral alumina for more than $1 \mathrm{~d}$.

General Procedure for Catalytic Site-Selective Acylation of 1 (Tables 1, 2) $\mathbf{1}$ (1.0 eq), $\mathbf{6}(10 \mathrm{~mol} \%)$ and 2,4,6-collidine (1.5 eq) were dissolved in $\mathrm{CHCl}_{3}$ at $20^{\circ} \mathrm{C}$. After the mixture was cooled to $-20^{\circ} \mathrm{C}$, an acid anhydride (1.1 eq) was added to the mixture. After stirred at the same temperature for the period indicated in Tables 1 and 2, the reaction was quenched with $\mathrm{MeOH}$. The mixture was diluted with AcOEt and washed with $1 \mathrm{M} \mathrm{HCl}$, saturated aq. $\mathrm{NaHCO}_{3}$, and brine, dried over $\mathrm{Na}_{2} \mathrm{SO}_{4}$, filtered and concentrated in vacuo. The residue was purified by preparative TLC $\left(\mathrm{MeOH}-\mathrm{CHCl}_{3}=5: 95\right)$ to give a mixture of 7-O- and 10-O-acylated products. The siteselectivity of the reaction was determined by ${ }^{1} \mathrm{H}-\mathrm{NMR}$ integration. This mixture was further purified by preparative TLC (AcOEt-hexane $=80: 20$ ) to give the 10 - $O$-acylated product.

10-Isobutyloyl 10-Deacetylbaccatin III (1b)

Colorless oil. $[\alpha]_{\mathrm{D}}^{20}-89 \quad\left(c=0.64, \quad \mathrm{CHCl}_{3}\right) . \quad{ }^{1} \mathrm{H}-\mathrm{NMR}$ $\left(400 \mathrm{MHz}, \mathrm{CDCl}_{3}\right) \delta: 8.10(\mathrm{dd}, J=8.4,0.8 \mathrm{~Hz}, 2 \mathrm{H}), 7.61(\mathrm{t}$, $J=7.2 \mathrm{~Hz}, 1 \mathrm{H}), 7.48(\mathrm{t}, J=7.6 \mathrm{~Hz}, 2 \mathrm{H}), 6.30(\mathrm{~s}, 1 \mathrm{H}), 5.62(\mathrm{~d}$, $J=6.8 \mathrm{~Hz}, 1 \mathrm{H}), 4.98(\mathrm{dd}, J=9.6,2.4 \mathrm{~Hz}, 1 \mathrm{H}), 4.88(\mathrm{t}, J=7.6 \mathrm{~Hz}$, $1 \mathrm{H}), 4.47(\mathrm{dd}, J=10.4,6.8 \mathrm{~Hz}, 1 \mathrm{H}), 4.30(\mathrm{~d}, J=8.4 \mathrm{~Hz}, 1 \mathrm{H})$, $4.14(\mathrm{~d}, J=8.4 \mathrm{~Hz} 1 \mathrm{H}), 3.88$ (d, $J=7.2 \mathrm{~Hz}, 1 \mathrm{H}), 2.73$ (heptet, $J=6.8 \mathrm{~Hz}, 1 \mathrm{H}), 2.66-2.50(\mathrm{~m}, 2 \mathrm{H}), 2.32-2.26(\mathrm{~m}, 5 \mathrm{H})$, 2.20 (brs, 1H), $2.04(\mathrm{~s}, 3 \mathrm{H}), 1.91-1.80(\mathrm{~m}, 1 \mathrm{H}), 1.66(\mathrm{~s}, 3 \mathrm{H})$, $1.32(\mathrm{~d}, J=6.8 \mathrm{~Hz}, 3 \mathrm{H}), 1.24(\mathrm{~d}, J=7.2 \mathrm{~Hz}, 3 \mathrm{H}), 1.10(\mathrm{~s}, 6 \mathrm{H})$. ${ }^{13} \mathrm{C}-\mathrm{NMR}\left(100 \mathrm{MHz}, \mathrm{CDCl}_{3}\right) \quad \delta: 204.2,177.2,170.6,167.0$, $146.2,133.7,131.9,130.1,129.2,128.6,84.4,80.7,79.1,76.4$, $75.8,74.9,72.3,67.9,58.6,46.1,42.6,38.5,35.5,34.0,26.9$, 22.6, 20.9, 19.2, 18.6, 15.6, 9.4 IR (neat) 3485, 2976, 2941, $1715,1452 \mathrm{~cm}^{-1}$. MS (FAB) $\mathrm{m} / \mathrm{z}$ (rel intensity) $615\left(\mathrm{M}+\mathrm{H}^{+}, 2\right)$, 252 (60), 73 (100). High resolution (HR)-MS (FAB) $\mathrm{m} / z$ Calcd for $\mathrm{C}_{33} \mathrm{H}_{43} \mathrm{O}_{11}(\mathrm{M}+\mathrm{H})^{+}$615.2805. Found 615.2800.

Baccatin III (2)

Colorless amorphous. $[\alpha]_{\mathrm{D}}^{20}-77\left(c=0.36, \mathrm{CHCl}_{3}\right) .{ }^{1} \mathrm{H}-\mathrm{NMR}$ $\left(400 \mathrm{MHz}, \mathrm{CDCl}_{3}\right) \delta: 8.10(\mathrm{dd}, J=8.8,1.6 \mathrm{~Hz}, 2 \mathrm{H}), 7.61(\mathrm{t}$, $J=7.6 \mathrm{~Hz}, 1 \mathrm{H}), 7.48(\mathrm{t}, J=7.6 \mathrm{~Hz}, 2 \mathrm{H}), 6.32(\mathrm{~s}, 1 \mathrm{H}), 5.62(\mathrm{~d}$, $J=7.6 \mathrm{~Hz}, 1 \mathrm{H}), 4.98(\mathrm{dd}, J=9.6,2.0 \mathrm{~Hz}, 1 \mathrm{H}), 4.90(\mathrm{t}, J=7.4 \mathrm{~Hz}$, $1 \mathrm{H}), 4.47$ (dd, $J=11.2,6.8 \mathrm{~Hz}, 1 \mathrm{H}), 4.31$ (d, $J=8.0 \mathrm{~Hz}, 1 \mathrm{H}), 4.15$ $(\mathrm{d}, J=8.0 \mathrm{~Hz} 1 \mathrm{H}), 3.88(\mathrm{~d}, J=6.8 \mathrm{~Hz}, 1 \mathrm{H}), 2.61-2.51(\mathrm{~m}, 1 \mathrm{H})$, 2.30-2.27 (m, 5H), $2.24(\mathrm{~s}, 3 \mathrm{H}), 2.05(\mathrm{~s}, 3 \mathrm{H}), 1.91-1.81(\mathrm{~m}$, $1 \mathrm{H}), 1.67$ (s, 3H), $1.10(\mathrm{~s}, 6 \mathrm{H}) .{ }^{13} \mathrm{C}-\mathrm{NMR}\left(100 \mathrm{MHz}, \mathrm{CDCl}_{3}\right)$ $\delta: 204.2,171.4,170.6,167.0,146.4,133.7,131.8,130.1,129.2$, 128.6, 84.4, 80.7, 79.1, 76.4, 76.2, 74.8, 72.3, 67.9, 58.7, 46.1, 42.7, 38.5, 35.6, 26.9, 22.6, 20.9, 15.6, 9.4 IR (neat) 3515, 2943, $1716,1241,1071,756 \mathrm{~cm}^{-1}$. MS (FAB) $\mathrm{m} / z$ (rel intensity) 609 $\left(\mathrm{M}+\mathrm{Na}^{+}, 10\right), 587\left(\mathrm{M}+\mathrm{H}^{+}, 2\right), 154$ (100). HR-MS (FAB) $\mathrm{m} / \mathrm{z}$ Calcd for $\mathrm{C}_{31} \mathrm{H}_{38} \mathrm{O}_{11} \mathrm{Na}(\mathrm{M}+\mathrm{Na})^{+}$609.2312. Found 609.2315.

10-O-Lauryl-10-deacetylbaccatin III

Colorless amorphous. $[\alpha]_{\mathrm{D}}^{20}-54\left(c=0.75, \mathrm{CHCl}_{3}\right) .{ }^{1} \mathrm{H}-\mathrm{NMR}$ $\left(400 \mathrm{MHz}, \mathrm{CDCl}_{3}\right) \delta: 8.10(\mathrm{dd}, J=7.2,1.6 \mathrm{~Hz}, 2 \mathrm{H}), 7.61(\mathrm{t}$, $J=7.4 \mathrm{~Hz}, 1 \mathrm{H}), 7.48$ (t, $J=7.8 \mathrm{~Hz}, 2 \mathrm{H}), 6.31$ (s, 1H), 5.62 (d, $J=6.8 \mathrm{~Hz}, 1 \mathrm{H}), 4.98$ (d, $J=7.6 \mathrm{~Hz}, 1 \mathrm{H}), 4.89$ (brt, $1 \mathrm{H}$ ), $4.50-4.44(\mathrm{~m}, 1 \mathrm{H}), 4.31(\mathrm{~d}, J=8.4 \mathrm{~Hz}, 1 \mathrm{H}), 4.15(\mathrm{~d}, J=8.4 \mathrm{~Hz}$, $1 \mathrm{H}), 3.88(\mathrm{~d}, J=6.8 \mathrm{~Hz}, 1 \mathrm{H}), 2.62-2.40$ (m, 4H), 2.34-2.22 (m, 5H), 2.14 (brd, 1H), 2.04 (s, 3H), 1.91-1.81 (m, 1H), 1.71 (quint, $J=7.0 \mathrm{~Hz}, 2 \mathrm{H}), 1.66(\mathrm{~s}, 3 \mathrm{H}), 1.44-1.18$ (br, $16 \mathrm{H}), 1.10$ $(\mathrm{s}, 6 \mathrm{H}), 0.88(\mathrm{t}, J=7.0 \mathrm{~Hz}, 3 \mathrm{H}) \cdot{ }^{13} \mathrm{C}-\mathrm{NMR}\left(100 \mathrm{MHz}, \mathrm{CDCl}_{3}\right)$ $\delta: 204.2,174.1,170.6,167.0,146.2,133.7,131.9,130.1,129.3$, $128.6,84.5,80.8,79.1,76.4,75.9,74.9,72.3,68.0,58.7,46.1$, $42.7,38.5,35.5,34.2,31.9,29.6,29.5,29.3,29.2,29.1,27.0$, 24.8, 22.7, 22.6, 20.9, 15.6, 14.1, 9.4. IR (neat) 3505, 2925, $1715,1240 \mathrm{~cm}^{-1}$. MS (FAB) $\mathrm{m} / z$ (rel intensity) $749\left(\mathrm{M}+\mathrm{Na}^{+}\right.$, 60), $727\left(\mathrm{M}+\mathrm{H}^{+}, 3\right), 176$ (100). HR-MS (FAB) $\mathrm{m} / \mathrm{z}$ Calcd for $\mathrm{C}_{41} \mathrm{H}_{58} \mathrm{O}_{11} \mathrm{Na}(\mathrm{M}+\mathrm{Na})^{+}$749.3877. Found 749.3878.

10-O-Benzoyl-10-deacetylbaccatin III

Colorless powder. mp $179-181^{\circ} \mathrm{C}$. $[\alpha]_{\mathrm{D}}^{20}-63(c=1.3$, THF). ${ }^{1} \mathrm{H}-\mathrm{NMR}\left(400 \mathrm{MHz}, \mathrm{CDCl}_{3}\right) \delta: 8.13-8.07(\mathrm{~m}, 4 \mathrm{H}), 7.62(\mathrm{t}$, 
$J=7.0 \mathrm{~Hz}, 2 \mathrm{H}), 7.49$ (t, $J=7.8 \mathrm{~Hz}, 2 \mathrm{H}), 7.48(\mathrm{t}, J=7.8 \mathrm{~Hz}, 2 \mathrm{H})$, $6.59(\mathrm{~s}, 1 \mathrm{H}), 5.67(\mathrm{~d}, J=7.2 \mathrm{~Hz}, 1 \mathrm{H}), 5.01(\mathrm{~d}, J=8.0 \mathrm{~Hz}, 1 \mathrm{H})$, $4.93(\mathrm{t}, J=7.8 \mathrm{~Hz}, 1 \mathrm{H}), 4.60-4.52(\mathrm{~m}, 1 \mathrm{H}), 4.33(\mathrm{~d}, J=8.4 \mathrm{~Hz}$, $1 \mathrm{H}), 4.17(\mathrm{~d}, J=8.4 \mathrm{~Hz}, 1 \mathrm{H}), 3.96(\mathrm{~d}, J=6.8 \mathrm{~Hz}, 1 \mathrm{H}), 2.71-2.55$ $(\mathrm{m}, 2 \mathrm{H}), 2.39-2.26(\mathrm{~m}, 5 \mathrm{H}), 2.16$ (brs, 1H), $2.11(\mathrm{~s}, 3 \mathrm{H})$, 1.95-1.84 (m, 1H), 1.693 (s, 3H), 1.690 (brs, 1H), 1.23 (s, $3 \mathrm{H}), 1.19(\mathrm{~s}, 3 \mathrm{H}) .{ }^{13} \mathrm{C}-\mathrm{NMR}\left(100 \mathrm{MHz}, \mathrm{CDCl}_{3}\right) \delta: 204.0,170.7$, $167.1,166.4,146.6,133.7,133.6,131.8,130.1,130.0,129.25$, $129.17,128.6,128.5,84.5,80.8,79.1,76.6,76.4,74.9,72.4$, $68.0,58.7,46.2,42.7,38.5,35.7,27.2,22.6,21.2,15.7,9.4$. IR (neat) $3483,2942,1717,1451,1270 \mathrm{~cm}^{-1}$. MS (FAB) $\mathrm{m} / \mathrm{z}$ (rel intensity) $671\left(\mathrm{M}+\mathrm{Na}^{+}, 2\right), 154$ (100). HR-MS (FAB) $\mathrm{m} / \mathrm{z}$ Calcd for $\mathrm{C}_{36} \mathrm{H}_{40} \mathrm{O}_{11} \mathrm{Na}(\mathrm{M}+\mathrm{Na})^{+}$671.2468. Found 671.2462.

10-O-Cinnamoyl-10-deacetylbaccatin III

Colorless microcrystal. mp $152-157^{\circ} \mathrm{C}$. $[\alpha]_{\mathrm{D}}^{20}-79(c=0.70$, $\left.\mathrm{CHCl}_{3}\right) .{ }^{1} \mathrm{H}-\mathrm{NMR}\left(400 \mathrm{MHz}, \mathrm{CDCl}_{3}\right) \quad \delta: 8.11$ (dd, $J=7.2$, $1.2 \mathrm{~Hz}, 2 \mathrm{H}), 7.78$ (d, $J=16.4 \mathrm{~Hz}, 1 \mathrm{H}), 7.65-7.53(\mathrm{~m}, 3 \mathrm{H}), 7.49$ $(\mathrm{t}, J=7.8 \mathrm{~Hz}, 2 \mathrm{H}), 7.44-7.37(\mathrm{~m}, 3 \mathrm{H}), 6.59(\mathrm{~d}, J=15.6 \mathrm{~Hz}, 1 \mathrm{H})$, $6.47(\mathrm{~s}, 1 \mathrm{H}), 5.65(\mathrm{~d}, J=7.2 \mathrm{~Hz}, 1 \mathrm{H}), 5.01(\mathrm{~d}, J=7.6 \mathrm{~Hz}, 1 \mathrm{H})$, 4.92 (brt, 1H), 4.56-4.50 (m, 1H), 4.32 (d, $J=8.4 \mathrm{~Hz}, 1 \mathrm{H}), 4.17$ (d, $J=8.0 \mathrm{~Hz}, 1 \mathrm{H}), 3.92$ (d, $J=7.2 \mathrm{~Hz}, 1 \mathrm{H}), 2.73$ (d, $J=4.0 \mathrm{~Hz}$, $1 \mathrm{H}), 2.65-2.53(\mathrm{~m}, 1 \mathrm{H}), 2.34-2.25(\mathrm{~m}, 5 \mathrm{H}), 2.18(\mathrm{brd}, 1 \mathrm{H})$, 2.09 (s, 3H), 1.94-1.83 (m, 1H), 1.69 (s, 3H), 1.67 (s, 1H), 1.17 $(\mathrm{s}, 3 \mathrm{H}), 1.16(\mathrm{~s}, 3 \mathrm{H}) \cdot{ }^{13} \mathrm{C}-\mathrm{NMR}\left(100 \mathrm{MHz}, \mathrm{CDCl}_{3}\right) \delta: 204.2$, $170.7,167.1,166.9,146.7,146.6,134.1,133.7,131.9,130.7$, $130.1,129.3,128.9,128.6,128.3,116.9,84.5,80.8,79.1,76.4$, $76.2,74.9,72.4,68.0,58.7,46.1,42.7,38.5,35.6,27.1,22.6$, 21.1, 15.7, 9.4. IR (neat) $3523,2942,1715,1633,1452 \mathrm{~cm}^{-1}$. MS (FAB) $m / z$ (rel intensity) $697\left(\mathrm{M}+\mathrm{Na}^{+}, 2\right), 676(\mathrm{M}+\mathrm{H}, 1)$, 154 (100). HR-MS (FAB) $m / z$ Calcd for $\mathrm{C}_{38} \mathrm{H}_{42} \mathrm{O}_{11} \mathrm{Na}(\mathrm{M}+\mathrm{Na})^{+}$ 697.2625. Found 697.2632.

10-O-2-Thiophenyl-10-deacetylbaccatin III

Colorless powder. mp $175-179^{\circ} \mathrm{C} . \quad[\alpha]_{\mathrm{D}}^{20}-60 \quad(c=0.55$, THF). ${ }^{1} \mathrm{H}-\mathrm{NMR}\left(400 \mathrm{MHz}, \mathrm{CDCl}_{3}\right) \delta: 8.11(\mathrm{dd}, J=7.6,1.2 \mathrm{~Hz}$, 2H), 7.89 (dd, $J=7.6,1.2 \mathrm{~Hz}, 1 \mathrm{H}), 7.65-7.57(\mathrm{~m}, 2 \mathrm{H}), 7.49$ $(\mathrm{t}, J=7.8 \mathrm{~Hz}, 2 \mathrm{H}), 7.15(\mathrm{dd}, J=5.0,3.8 \mathrm{~Hz}, 1 \mathrm{H}), 6.53(\mathrm{~s}, 1 \mathrm{H})$, $5.66(\mathrm{~d}, J=6.8 \mathrm{~Hz}, 1 \mathrm{H}), 5.00(\mathrm{~d}, J=8.0 \mathrm{~Hz}, 1 \mathrm{H}), 4.92$ (brt, $1 \mathrm{H}), 4.55-4.48(\mathrm{~m}, 1 \mathrm{H}), 4.32(\mathrm{~d}, J=8.4 \mathrm{~Hz}, 1 \mathrm{H}), 4.16(\mathrm{~d}$, $J=8.4 \mathrm{~Hz}, 1 \mathrm{H}), 3.94(\mathrm{~d}, J=6.8 \mathrm{~Hz}, 1 \mathrm{H}), 2.67-2.54(\mathrm{~m}, 1 \mathrm{H})$, $2.50(\mathrm{~d}, J=4.4 \mathrm{~Hz}, 1 \mathrm{H}), 2.39-2.25(\mathrm{~m}, 5 \mathrm{H}), 2.20-2.08(\mathrm{~m}$, $4 \mathrm{H}), 1.93-1.82(\mathrm{~m}, 1 \mathrm{H}), 1.69(\mathrm{~s}, 3 \mathrm{H}), 1.66(\mathrm{~s}, 1 \mathrm{H}), 1.20(\mathrm{~s}$, $3 \mathrm{H}), 1.17(\mathrm{~s}, 3 \mathrm{H}) .{ }^{13} \mathrm{C}-\mathrm{NMR}\left(100 \mathrm{MHz}, \mathrm{CDCl}_{3}\right) \delta: 203.9,170.7$, $167.1,161.9,146.5,134.5,133.7,133.4,132.5,131.6,130.1$, $129.2,128.6,128.0,84.4,80.8,79.1,76.5,76.4,74.8,72.3$, 68.0, 58.7, 46.2, 42.7, 38.5, 35.7, 27.0, 22.6, 21.0, 15.7, 9.4. IR (neat) $3477,2937,1715,1259 \mathrm{~cm}^{-1}$. MS (FAB) $\mathrm{m} / z$ (rel intensity) $677\left(\mathrm{M}+\mathrm{Na}^{+}, 5\right), 154$ (100). HR-MS (FAB) $\mathrm{m} / \mathrm{z}$ Calcd for $\mathrm{C}_{34} \mathrm{H}_{38} \mathrm{O}_{11} \mathrm{SNa}(\mathrm{M}+\mathrm{Na})^{+}$677.2033. Found 677.2032.

10-O-3-Furyl-10-deacetylbaccatin III

Colorless powder. mp $155-160^{\circ} \mathrm{C} .[\alpha]_{\mathrm{D}}^{20}-83(c=0.75$, THF $)$. ${ }^{1} \mathrm{H}-\mathrm{NMR}\left(400 \mathrm{MHz}, \mathrm{CDCl}_{3}\right) \delta: 8.14-8.08(\mathrm{~m}, 3 \mathrm{H}), 7.62(\mathrm{t}$, $J=7.6 \mathrm{~Hz}, 1 \mathrm{H}), 7.52-7.46(\mathrm{~m}, 3 \mathrm{H}), 6.78(\mathrm{~d}, J=1.2 \mathrm{~Hz}, 1 \mathrm{H})$, $6.51(\mathrm{~s}, 1 \mathrm{H}), 5.65(\mathrm{~d}, J=7.2 \mathrm{~Hz}, 1 \mathrm{H}), 5.00(\mathrm{~d}, J=8.0 \mathrm{~Hz}, 1 \mathrm{H})$, 4.92 (brq, 1H), 4.57-4.47 (m, 1H), 4.32 (d, $J=8.4 \mathrm{~Hz}, 1 \mathrm{H})$, $4.16(\mathrm{~d}, J=8.4 \mathrm{~Hz}, 1 \mathrm{H}), 3.93(\mathrm{~d}, J=6.8 \mathrm{~Hz}, 1 \mathrm{H}), 2.65-2.52$ (m, $2 \mathrm{H}), 2.36-2.26(\mathrm{~m}, 5 \mathrm{H}), 2.11$ (brs, 1H), $2.10(\mathrm{~s}, 3 \mathrm{H}), 1.94-1.82$ $(\mathrm{m}, 1 \mathrm{H}), 1.69(\mathrm{~s}, 3 \mathrm{H}), 1.65(\mathrm{~s}, 1 \mathrm{H}), 1.17(\mathrm{~s}, 3 \mathrm{H}), 1.15(\mathrm{~s}, 3 \mathrm{H})$. ${ }^{13} \mathrm{C}-\mathrm{NMR}\left(100 \mathrm{MHz}, \mathrm{CDCl}_{3}\right) \delta$ : 204.0, 170.7, 167.1, 162.8, $148.5,146.5,144.0,133.7,131.7,130.1,129.2,128.6,118.5$, $109.9,84.4,80.8,79.1,76.4,76.1,74.8,72.3,68.0,58.7,46.2$, $42.7,38.5,35.7,27.1,22.6,21.1,15.7,9.4$. IR (neat) 3505, 2943,
1714, $1272 \mathrm{~cm}^{-1}$. MS (FAB) $\mathrm{m} / z$ (rel intensity) $661\left(\mathrm{M}+\mathrm{Na}^{+}\right.$, 10), $639\left(\mathrm{M}+\mathrm{H}^{+}, 1\right), 154$ (100). HR-MS (FAB) $\mathrm{m} / \mathrm{z}$ Calcd for $\mathrm{C}_{34} \mathrm{H}_{38} \mathrm{O}_{12} \mathrm{Na}(\mathrm{M}+\mathrm{Na})^{+}$661.2261. Found 661.2264.

7-O-Trichloroacetyl-10-deacetylbaccatin III

Colorless needle. mp $218^{\circ} \mathrm{C}$ (dec.). $[\alpha]_{\mathrm{D}}^{20}-27 \quad(c=0.50$, $\left.\mathrm{CHCl}_{3}\right) .{ }^{1} \mathrm{H}-\mathrm{NMR}\left(400 \mathrm{MHz}, \mathrm{CDCl}_{3}\right) \quad \delta: 8.10 \quad$ (dd, $J=8.4$, $2.4 \mathrm{~Hz}, 2 \mathrm{H}), 7.62(\mathrm{t}, J=7.2 \mathrm{~Hz}, 1 \mathrm{H}), 7.49(\mathrm{t}, J=7.6 \mathrm{~Hz}, 2 \mathrm{H})$, $5.65(\mathrm{~d}, J=6.8 \mathrm{~Hz}, 1 \mathrm{H}), 5.56(\mathrm{dd}, J=10.4,7.2 \mathrm{~Hz}, 1 \mathrm{H}), 5.32$ (s, 1H), 4.99 (d, $J=8.4 \mathrm{~Hz}, 1 \mathrm{H}), 4.88(\mathrm{t}, J=7.6 \mathrm{~Hz}, 1 \mathrm{H}), 4.35$ $(\mathrm{d}, J=8.8 \mathrm{~Hz}, 1 \mathrm{H}), 4.19(\mathrm{~d}, J=8.0 \mathrm{~Hz}, 1 \mathrm{H}), 4.10(\mathrm{~d}, J=6.8 \mathrm{~Hz}$, $1 \mathrm{H}), 3.99$ (brs, 1H), 2.78-2.65 (m, 1H), 2.34-2.23 (m, 5H), $2.12(\mathrm{~s}, 3 \mathrm{H}), 2.10-2.00(\mathrm{~m}, 2 \mathrm{H}), 1.92(\mathrm{~s}, 3 \mathrm{H}), 1.08(\mathrm{~s}, 3 \mathrm{H}), 1.07$ $(\mathrm{s}, 3 \mathrm{H}),{ }^{13} \mathrm{C}-\mathrm{NMR}\left(100 \mathrm{MHz}, \mathrm{CDCl}_{3}\right) \delta: 209.9,170.9,166.9$, 161.0, 142.8, 134.3, 133.8, 130.1, 129.1, 128.7, 83.3, 80.1, 78.7, $77.9,76.4,74.9,74.3,67.8,56.1,46.7,42.5,38.5,32.5,26.6$, $22.5,19.4,15.0,10.8$. IR (neat) $3466,2949,1767,1715,1452$, $1244 \mathrm{~cm}^{-1}$. MS (FAB) $\mathrm{m} / z$ (rel intensity) $689\left(\mathrm{M}+\mathrm{H}^{+}, 2\right), 185$ (60), 105 (100). HR-MS (FAB) $m / z$ Calcd for $\mathrm{C}_{31} \mathrm{H}_{36} \mathrm{O}_{11} \mathrm{Cl}_{3}$ $(\mathrm{M}+\mathrm{H})^{+}$689.1323. Found 689.1310.

10-Deacetylbaccatin III Dimer 12

To a solution of pivaloyl chloride $(28 \mu \mathrm{L}, 230 \mu \mathrm{mol}, 2.5 \mathrm{eq})$ was added undecanedioic acid $(10 \mathrm{mg}, 46 \mu \mathrm{mol}, 0.5 \mathrm{eq})$ and DIPEA $(47 \mu \mathrm{L}, 347 \mu \mathrm{mol}, 3.8 \mathrm{eq})$ in $\mathrm{CH}_{2} \mathrm{Cl}_{2}(1.0 \mathrm{~mL})$ dropwise at room temperature, and the mixture was stirred at room temperature for $2 \mathrm{~h}$. The resulting mixture was concentrated in vacuo and diluted with $\mathrm{CHCl}_{3}(1.0 \mathrm{~mL})$. The resulting mixture was added to a precooled solution of $1(50 \mathrm{mg}, 92 \mu \mathrm{mol}$, $1.0 \mathrm{eq}), 6(7.6 \mathrm{mg}, 9.2 \mu \mathrm{mol}, 10 \mathrm{~mol} \%)$ and collidine $(37 \mu \mathrm{g}$, $280 \mu \mathrm{mol}, 3.0 \mathrm{eq})$ in $\mathrm{CHCl}_{3}(8.0 \mathrm{~mL}$, total $9.0 \mathrm{~mL})$ at $-20^{\circ} \mathrm{C}$ (final concentration of 1: $0.01 \mathrm{M}$ ). After stirred at $-20^{\circ} \mathrm{C}$ for $30 \mathrm{~h}$, the reaction mixture was quenched with methanol $(5.0 \mathrm{~mL})$ and stired at room temperature for $1 \mathrm{~h}$. The mixture was diluted with AcOEt and washed with $1 \mathrm{M} \mathrm{HCl}$, saturated aq. $\mathrm{NaHCO}_{3}$, and brine, dried over $\mathrm{Na}_{2} \mathrm{SO}_{4}$, filtered and concentrated in vacuo. The residue was purified by preparative TLC (AcOEt-hexane $=80: 20$ ) to give a 10-deacetylbaccatin III dimer 12 as colorless amorphous $(23 \mathrm{mg}, 40 \%$ yield as a single isomer). $[\alpha]_{\mathrm{D}}^{20}-75(c=1.0, \mathrm{THF}) .{ }^{1} \mathrm{H}-\mathrm{NMR}(400 \mathrm{MHz}$, $\left.\mathrm{CDCl}_{3}\right) \delta: 8.06(\mathrm{dd}, J=8.4,1.2 \mathrm{~Hz}, 4 \mathrm{H}), 7.58(\mathrm{t}, J=7.6 \mathrm{~Hz}$, $2 \mathrm{H}), 7.45(\mathrm{t}, J=7.8 \mathrm{~Hz}, 4 \mathrm{H}), 6.31(\mathrm{~s}, 2 \mathrm{H}), 5.60(\mathrm{~d}, J=7.2 \mathrm{~Hz}$, $2 \mathrm{H}), 4.98(\mathrm{~d}, J=7.6 \mathrm{~Hz}, 2 \mathrm{H}), 4.88(\mathrm{t}, J=7.8 \mathrm{~Hz}, 2 \mathrm{H}), 4.47$ (dd, $J=10.4,6.8 \mathrm{~Hz}, 2 \mathrm{H}), 4.29$ (d, $J=7.2 \mathrm{~Hz}, 2 \mathrm{H}), 4.14$ (d, $J=8.4 \mathrm{~Hz}$, $2 \mathrm{H}), 3.87$ (d, $J=7.2 \mathrm{~Hz}, 2 \mathrm{H}), 2.68$ (brs, 2H), 2.59-2.41 (m, $6 \mathrm{H}), 2.32-2.26(\mathrm{~m}, 10 \mathrm{H}), 2.04(\mathrm{~s}, 6 \mathrm{H}), 1.90-1.76(\mathrm{~m}, 4 \mathrm{H}), 1.71$ (quint, $J=7.2 \mathrm{~Hz}, 4 \mathrm{H}), 1.65(\mathrm{~s}, 6 \mathrm{H}), 1.45-1.30(\mathrm{~m}, 10 \mathrm{H}), 1.12(\mathrm{~s}$, $6 \mathrm{H}), 1.10(\mathrm{~s}, 6 \mathrm{H}) .{ }^{13} \mathrm{C}-\mathrm{NMR}\left(100 \mathrm{MHz}, \mathrm{CDCl}_{3}\right) \delta: 204.2,174.1$, $170.6,167.0,146.5,133.6,131.8,130.1,129.3,128.6,84.4,80.7$, $79.0,76.4,76.0,74.9,72.3,67.8,58.6,46.1,42.6,38.7,35.5$, $34.2,29.0,28.8,27.0,24.8,22.6,21.0,15.6,9.4$. IR (neat) 3503, 2932, 1716, 1242, $1070 \mathrm{~cm}^{-1}$. MS (FAB) $\mathrm{m} / z$ (rel intensity) $1292\left(\mathrm{M}+\mathrm{Na}^{+}, 2\right), 154$ (100). HR-MS (FAB) $\mathrm{m} / \mathrm{z}$ Calcd for $\mathrm{C}_{69} \mathrm{H}_{88} \mathrm{O}_{22} \mathrm{Na}(\mathrm{M}+\mathrm{Na})^{+}$1291.5665. Found 1291.5660.

Acknowledgments This reseach was financially supported by a Grant-in-Aid for Scientific Research on Innovative Areas "Advanced Molecular Transformations by Organocatalysts" and Scientific Research (S) from the Ministry of Education, Culture, Sports, Science and Technology (MEXT) of Japan.

Conflict of Interest The authors declare no conflict of interest. 
Supplementary Materials The online version of this article contains supplementary materials.

\section{References and Note}

1) Newman D. J., Cragg G. M., J. Nat. Prod., 75, 311-335 (2012).

2) González-Sabín J., Morán-Ramallal R., Rebolledo F., Chem. Soc. Rev., 40, 5321-5335 (2011).

3) Gu J., Ruppen M. E., Cai P., Org. Lett., 7, 3945-3948 (2005).

4) Robles O., Romo D., Nat. Prod. Rep., 31, 318-334 (2014).

5) Kawabata T., Muramatsu W., Nishio T., Shibata T., Schedel H., J. Am. Chem. Soc., 129, 12890-12895 (2007).

6) Ueda Y., Muramatsu E., Mishiro K., Furuta T., Kawabata T., J. Org. Chem., 74, 8802-8805 (2009).

7) Yoshida K., Furuta T., Kawabata T., Tetrahedron Lett., 51, 48304832 (2010).

8) Ueda Y., Mishiro K., Yoshida K., Furuta T., Kawabata T., J. Org. Chem., 77, 7850-7857 (2012).

9) Takeuchi H., Mishiro K., Ueda Y., Fujimori Y., Furuta T., Kawabata T., Angew. Chem. Int. Ed., 54, 6177-6180 (2015).

10) Ueda Y., Furuta T., Kawabata T., Angew. Chem. Int. Ed., 54, 1196611970 (2015).

11) El-Dakdouki M. H., Erhardt P. W., Pure Appl. Chem., 84, 1479 1542 (2012), and references cited therein.

12) Guéritte-Voegelein F., Sénilh V., David B., Guénard D., Potier P., Tetrahedron, 42, 4451-4460 (1986).

13) Denis J.-N., Greene A. E., Guénard D., Guéritte-Voegelein F., Mangatal L., Potier P., J. Am. Chem. Soc., 110, 5917-5919 (1988).

14) Holton R. A., Zhang Z., Clarke P. A., Nadizadeh H., Procter D. J.,
Tetrahedron Lett., 39, 2883-2886 (1998).

15) An alternative method using catalytic amount of lanthanide triflates as Lewis Acid has been reported. Damen E. W. P., Braamer L., Scheeren H. W., Tetrahedron Lett., 39, 6081-6082 (1998).

16) Xu S., Held I., Kempf B., Mayr H., Steglich W., Zipse H., Chem. Eur. J., 11, 4751-4757 (2005).

17) The similar phenomenon was observed in our previous study for acylation of carbohydrates, see, ref. 5 .

18) Lee D., Kim K.-C., Kim M.-J., Tetrahedron Lett., 39, 9039-9042 (1998).

19) Kim K. S., Chai K. B., Moon Y. H., Lee K. O., Kim N. D., Ha T. H., Shin J. A., Lee G. S., Kim W. J., U.S. Patent 6,130,336 (2000).

20) Sundram U. N., Griffin J. H., Nicas T. I., J. Am. Chem. Soc., 118, 13107-13108 (1996).

21) Matsumori N., Yamaji N., Matsuoka S., Oishi T., Murata M., J. Am. Chem. Soc., 124, 4180-4181 (2002).

22) Salunke D. B., Hazra B. G., Pore V. S., Bhat M. K., Nahar P. B., Deshpande M. V., J. Med. Chem., 47, 1591-1594 (2004).

23) Paz M. M., Suresh Kumar G., Glover M., Waring M. J., Tomasz M., J. Med. Chem., 47, 3308-3319 (2004).

24) Lu J., Yoshida O., Hayashi S., Arimoto H., Chem. Commun., 2007, 251-253 (2007)

25) Kumar S., Arya D. P., Bioorg. Med. Chem. Lett., 21, 4788-4792 (2011).

26) A mixed anhydride method was found applicable for site-selective diversification of polyol natural products, see ref. 8 .

27) Kingston D. G. I., J. Nat. Prod., 63, 726-734 (2000). 